analysis revealed four dimensions: Level of comfort with EHR; Concern over current ability to monitor medication adherence; Attitudes about value of DM applications; and Benefits vs cost of DM for payers. Women scored higher on attitudes about the value of digital applications $(\mathrm{p}<0.01)$. Providers who perceive nonadherence as costly, and those who believe DM could benefit providers and patients scored higher on the value of DM $(\mathrm{p}<.05)$. Those whose LSP focuses on improving efficiency and prescribers with a higher proportion of Medicaid/ uninsured patients displayed concern about their ability to monitor adherence $(\mathrm{p}<0.05)$. Willingness to be a Beta Test site for DM applications was positively correlated with concern about their ability to monitor adherence and attitudes about the value of DM ( $\mathrm{p}<0.01)$.

Conclusions. Prescriber characteristics including LSP, focus on healthcare costs, and attitudes toward DM may be related to adoption of the IEM platform. Those with more Medicaid/ uninsured patients were more concerned about ability to monitor adherence while those focused-on cost and benefit to providers and patients viewed DM as part of a solution for managing outcomes and cost. Overall, LSP, patient panel size by payer type, and focus on healthcare cost containment should be considered when developing IEM provider training materials.

Funding. Otsuka Pharmaceutical Development \& Commercialization, Inc.

\section{Xylological Variant of Reverse Fregoli Syndrome, Delusions of Being a Tree}

\author{
Harsimran Singh Bakhshi ${ }^{1}$ and Alan Richard Hirsch ${ }^{2}$ \\ ${ }^{1}$ Windsor University School of Medicine, Cayon, St. Kitts and Nevis, Caribbean, \\ and ${ }^{2}$ Smell and Taste Treatment and Research Foundation, Chicago, IL, USA
}

Presenting Author: Harsimran Singh Bakhshi replacement by a living non-animal object has not hitherto been reported. Body infestation with animate objects, as in Ekbom syndrome, only includes animals not botanicals (Chaudhary, 2019). This could be viewed as Reverse Inanimate Capgras Syndrome: instead of an imposter replacing a close friend, who then is inserted into the sufferer; a tree has replaced the sufferer. Peradventure, this may fit into the construct of Intermetamorphosis, a misidentification syndrome associated with the belief that individuals have transformed into other persons (Jariwala, 2017). Botanical Intermetamorphosis, the belief by the sufferer that the other individual is transformed from a person into a plant has not been described. Reverse Intermetamorphosis is the projection of an external individual into the person suffering or a syndrome of altered physical and psychological identities of the self (Silva, 1990). However, in this situation, the objects are all human or animate animals not botanicals. In Fregoli syndrome, there is an altered physical identity of others. In Reverse Fregoli syndrome, the sufferer assumes the physical but not the psychological identity of the stranger (Silva, 1990). But in this instance, the stranger is human as opposed to a plant life form. In the current case there is only altered physical identity (into a tree) not psychological identity. The current case may also be interpreted as a Botanical Variant of Interparietal Syndrome. In this condition, parts of the body are perceived to be lifeless, due to lesions of the inferior parietal lobe including supramarginalis gyrus, angular gyrus and the basalis parietalis area (Angyal, 1935). Investigation for those whom have Intermetamorphosis, Fregoli syndrome, Capgras syndrome, Interparietal syndrome, and Cotard's syndrome for the presence of delusions involving plant life is warranted.

\section{The Dilemma, Conversion Disorder or Stiff Person Syndrome, a Case Report}

\author{
Sultana Jahan, MD
}

University of Missouri, Department of Psychiatry, Columbia, MO, USA

Presenting Author: Sultana Jahan

\begin{abstract}
Study Objectives. The main objectives of this case study are 1. Clinicians facing symptoms that are difficult to interpret should exercise caution in diagnosing conversion disorder. 2. Increasing awareness about rare neurological conditions may appear as psychogenic illnesses. 3. Clinicians be advocate for their patients. Introduction. Conversion disorder is a mental condition in which a person present with one or more symptoms of altered voluntary motor or sensory function, or other neurologic symptoms that cannot be explained by medical evaluation. Stiff person syndrome (SPS) is a disabling autoimmune central nervous system disorder characterized by progressive muscle rigidity, gait impairment, with superimposed painful spasms. SPS is commonly associated with high anti-glutamic acid decarboxylase
\end{abstract}


(GAD) antibody titers. The dominant antigen recognized by these antibodies is the GABA-synthesizing enzyme GAD.

Method. Patient X, a 17-year-old Hispanic American female who presented to the Child and Adolescent Psychiatry Clinic with the complaint of ataxia \& aphasia associated with anxiety. Patient was referred by the neurology clinic after they could not establish any organic cause of her ataxia or aphasia. After thorough evaluation at the child psychiatry clinic she was given the diagnosis of anxiety secondary to ataxia and aphasia and r/o Conversion Disorder. She was initiated treatment with sertraline for her anxiety. Her sertraline dose was increased gradually up to $100 \mathrm{mg}$ daily. From the beginning the patient also received counseling \& physical therapy. With these combination of treatments, patient's symptoms did not get any better. Her symptoms actually got worse over time. At this point, the Child Psychiatry Clinic sent a message to the neurology clinic for further evaluation of patient due to her progressive gait and speech impairments.

Results. The neurology clinic saw the patient again and did further testing. The patient was positive for high titers of anti-glutamic acid decarboxylase antibodies (Anti-GAD). At this point, the patient was given the diagnosis of Stiff Person Syndrome. Patient was admitted to the hospital for further management. She was treated with benzodiazepines, IV immune globulin, \& steroid. Soon after discharge from the hospital, the patient was seen at the Child Psychiatric Clinic. The patient's mother reported, after the in-patient treatment, the patient's symptoms improved.

Discussion. It is essential for clinicians to look for neurologic \& other general medical conditions while evaluating a patient with possible conversion disorder. A systematic review of 27 studies found that among 1466 patients initially diagnosed with conversion symptoms, the frequency of misdiagnosis was approximately 4 percent. References. BMJ. 2005;331(7523):989. Epub Oct 13. Childhood onset of stiff-man syndrome. JAMA Neurol. 2013;70(12):1531. J Neurol Neurosurg Psychiatry. 2015 Aug; 86(8):840-8. Epub 2014 Dec 15.

\section{Assessing the Benefit-Risk Ratio of Approved Treatments for Bipolar Depression Using Likelihood to be Helped or Harmed (LHH) Analyses}

\author{
Leslie Citrome, MD, $\mathrm{MPH}^{1}$, Michael Tocco, $\mathrm{PhD}^{2}$, \\ Courtney Zeni, $\mathrm{PhD}^{2}$, Andrei Pikalov, MD, $\mathrm{PhD}^{2}$ and \\ Robert Goldman, PhD $^{2}$
}

${ }^{1}$ New York Medical College, Valhalla, NY, USA, and ${ }^{2}$ Sunovion Pharmaceuticals
Inc., Fort Lee, NJ and Marlborough, MA, USA

\section{Abstract}

Background. Four medications are FDA approved for bipolar depression: lurasidone (LUR), cariprazine (CAR), quetiapine IR \& XR (QUE), and olanzapine-fluoxetine combination (OFC). Indirect comparisons for efficacy using Number Needed to Treat (NNT) and for tolerability using Number Needed to Harm $(\mathrm{NNH})$ can be useful clinical benchmarks to aid treatment decisions. Benefit and risk may also be examined using the Likelihood to be Helped or Harmed (LHH). In this post-hoc analysis, we examined the benefit-risk ratio of the four treatments using LHH.

Method. Individual and pooled monotherapy data from shortterm clinical registration trials of patients with bipolar depression were assessed for LUR, CAR, pooled QUE (300 and $600 \mathrm{mg}$ ), and pooled OFC (considered as monotherapy for this study at fixed doses of $6 / 25,6 / 50,12 / 50 \mathrm{mg}$ ) data. NNT estimates were calculated using the proportions of MADRS responders (defined as $\geq$ $50 \%$ improvement at study endpoint) and MADRS remitters (defined as a score of $\leq 10$ [for LUR and CAR] and $\leq 12$ [for QUE and OFC]) at study endpoint. NNH data were calculated for the proportions of patients who discontinued due to an adverse event (AE) and for individual AEs commonly associated with each treatment. $\mathrm{LHH}$ was calculated as the ratio of $\mathrm{NNH} / \mathrm{NNT}$ to determine the benefit-risk ratio.

Results. The NNT estimates for response vs. placebo were: 5 for both LUR 20-60 mg and 80-120 mg; 10 for both CAR $1.5 \mathrm{mg}$ and $3.0 \mathrm{mg}$; 6 for QUE; and 4 for OFC. The NNTs for remission vs placebo were: 7 for LUR 20-60 mg and 9 for LUR $80-120 \mathrm{mg}$; 10 for CAR $1.5 \mathrm{mg}$ and 13 for CAR $3.0 \mathrm{mg}$; 6 for QUE; and 5 for OFC. The NNH estimates for discontinuations due to AEs were: 642 for LUR 20-60 mg and -151 for LUR 80-120 mg; 298 for CAR $1.5 \mathrm{mg}$ and 31 for CAR $3.0 \mathrm{mg}$; 10 for QUE; and -37 for OFC. NNH values that were negative were assigned a value of 1000 to permit LHH to be calculated. The LHHs for response vs discontinuation due to an AE were: 128.4 for LUR 20-60 mg and 200 for LUR 80-120 mg; 29.8 for CAR $1.5 \mathrm{mg}$ and 3.1 for CAR 3.0 mg; 1.7 for QUE; and 250 for OFC. The LHHs for response vs akathisia were: 3.6 for LUR 20-60 mg and 2.4 for LUR $80-120 \mathrm{mg}$; 3.6 for CAR $1.5 \mathrm{mg}$ and 1.3 for CAR $3.0 \mathrm{mg}$; 34 for QUE; and not available (NA) for OFC. The LHHs for response vs EPS were: 8 for LUR 20-60 mg and 3.2 for LUR 80-120 mg; 5 for CAR $1.5 \mathrm{mg}$ and 2.5 for CAR 3.0 mg; NA for QUE; and NA for OFC. The LHH for response vs weight gain was 5.8 for LUR $20-60 \mathrm{mg}$ and 1110 for LUR 80-120 mg; 5 for both doses of CAR; 2.7 for QUE; and 1.5 for OFC.

Conclusions. LHH can illustrate the trade-offs regarding potential benefits versus potential harms. Across a variety of measures, the lower-dose groups for both LUR and CAR generally evidenced a better benefit-risk profile than the higher-dose groups. While quetiapine and OFC demonstrated robust efficacy, their reduced tolerability resulted in a more marginal benefit-risk ratio for some of the outcomes.

Funding. Sunovion Pharmaceuticals Inc. 